REVIEW

\title{
Endometrial stromal tumours of the uterus: a practical approach using conventional morphology and ancillary techniques
}

\author{
Patricia Baker, Esther Oliva
}

J Clin Pathol 2007;60:235-243. doi: 10.1136/jcp.2005.031203

Endometrial stromal tumours (ESTs) are diagnosed in most instances by light microscopy. Often, the greatest challenge is to distinguish between the different subtypes of these tumours. Furthermore, a handful of new or relatively new entities have been described in the literature, which may cause problems in the differential diagnosis; highly cellular leiomyoma is the most common. In addition, new antibodies have been developed to help in the distinction of ESTs from their mimics, as there are prognostic and therapeutic implications. A practical approach is provided for the diagnosis of ESTs on the basis of systematic assessment of histological and immunohistochemical parameters, and recent developments related to these tumours are highlighted.

See end of article for authors' affiliations

......................

Correspondence to: E Oliva, Pathology Department (Warren 2) Massachusetts General Hospital, 55 Fruit Street, Boston, MA 02114 , USA; eoliva@partners.org

Accepted 16 January 2006

....................
E ndometrial stromal tumours (ESTs) of the uterus are the second most common mesenchymal tumours of the uterus even though they account for $<10 \%$ of all such tumours. ${ }^{1}$ In the latest 2003 World Health Organization classification, ${ }^{2}$ ESTs are divided into

a. endometrial stromal nodule (ESN),

b. low-grade endometrial stromal sarcoma (ESS),

c. undifferentiated endometrial sarcoma (UES).

The ESN and the low-grade ESS fall in the lower end of the spectrum of this group of tumours. Both are typically composed of a diffuse growth of small blue cells with scant cytoplasm, and oval to spindle nuclei that resemble the endometrial stromal cells of the proliferative endometrium (fig 1). ${ }^{3}$ At the other end of the spectrum is the UES, a very highgrade sarcoma, which does not resemble the proliferative endometrium. The diagnosis of UES is reached after excluding other high-grade tumours of the uterus with a sarcomatous component. $^{56}$

\section{DISTINCTION OF ESN FROM LOW-GRADE ESS}

Both tumours have similar presentation, vaginal bleeding being the most common. ${ }^{7}$ Of note, about a third to a half of the low-grade ESSs have extrauterine spread at the time of diagnosis and, rarely, these tumours may initially present at an extrauterine site, most commonly the ovary. ${ }^{6-9}$ Thus, when evaluating an ovarian tumour with a microscopic appearance consistent with an EST, it is important to exclude a history of a uterine EST and to suggest inspection of the uterus, as ESTs of the uterus are far more common than primary ovarian ESTs.

On gross examination, the main differentiating feature between the two neoplasms is tumour circumscription. Typically, ESN is a well-circumscribed, although non-encapsulated, neoplasm (fig 2). ${ }^{10}{ }^{11}$ In contrast, low-grade ESSs often show an irregular nodular growth affecting the endometrium, myometrium or both. The main mass is often associated with varying degrees of permeation of the myometrium, including worm-like plugs of tumour that fill and distend myometrial veins, often extending to parametrial veins. ${ }^{6}{ }^{12}$ However, on rare occasions, low-grade ESSs may appear deceptively well circumscribed on gross examination. Both tumours have a soft, tan to yellow cut surface.

Microscopically, the most important single criterion for the diagnosis of ESN is the finding of a non-infiltrative border of the tumour. Focal irregularities in the form of lobulated or finger-like projections into the adjacent myometrium that are not $\geqslant 3 \mathrm{~mm}$ and are not $>3$ in number may be seen. Vascular invasion is not allowed; thus, if present, the tumour should be diagnosed as a lowgrade ESS. ${ }^{10}$ In contrast with ESN, low-grade ESSs permeate the myometrium in irregular tongues and often invade myometrial (fig 3) as well as extrauterine veins and lymphatics (fig 4). ${ }^{6712}$ Myometrial invasion and vascular invasion are the two most important features used to distinguish between these two tumours. In most cases it is impossible to differentiate between an ESN and a low-grade ESS on the basis of curettage specimens and, thus, distinction can only be confidently established in a hysterectomy specimen. This is an important issue when the patient is of reproductive age and desires to preserve her uterus. In these circumstances, a combination of diagnostic imaging and hysteroscopy may be used to monitor the growth of the tumour, and occasionally local excision has been successful. ${ }^{13}{ }^{14}$

Abbreviations: ESN, endometrial stromal nodule; ESS, endometrial stromal sarcoma; EST, endometrial stromal tumour; EST-SMD, endometrial stromal tumours with smooth-muscle differentiation; HCL, highly cellular leimyoma; IVL, intravenous leiomyomatosis; PEComa, perivascular epithelioid cell; UES, undifferentiated endometrial sarcoma; UTROSCT, uterine tumour resembling an ovarian sex cord stromal tumour 


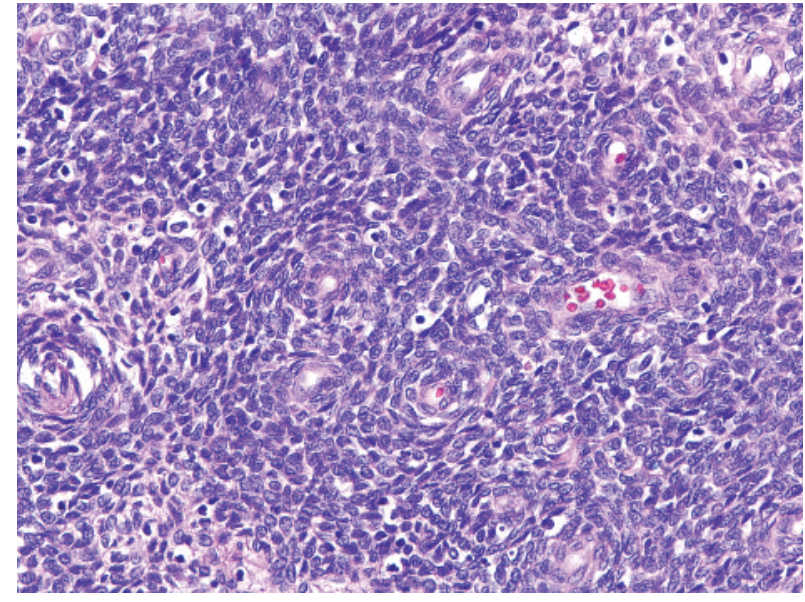

Figure 1 Endometrial stromal tumour. Small uniform cells with oval nuclei and scant cytoplasm grow in sheets and focally whorl around arterioles.

Other microscopic features, including whorling of the neoplastic stromal cells around arterioles, hyalinisation of the arteriolar walls, collagen bands or plaques, diffuse areas of hyalinisation, foamy histiocytes, cystic degeneration associated with cholesterol clefts, and necrosis, may be seen in both tumours and are not useful in the differential diagnosis. ${ }^{6}{ }^{10-12}$

The treatment of choice for an ESN is surgical resection, whereas patients with low-grade ESS undergo hysterectomy with bilateral salpingo-oophorectomy. Adjuvant treatment either with progestins, radiation therapy or even aromatase inhibitors may be given, depending on the extension of the tumour and patients' risk factors. ${ }^{15-20}$ From the prognostic point of view, it is extremely important to distinguish between these two tumours, as ESNs do not relapse and low-grade ESSs have a low malignant potential characterised by late recurrences. For this reason, patients with low-grade ESS should be followed up for an extended period of time, up to 30 years. ${ }^{67162122}$

\section{Potential pitfall}

Low-grade ESSs may be well circumscribed and may simulate an ESN on gross examination.

\section{Recommendations}

In endometrial curettage, the working diagnosis should be EST, as in most cases the margin cannot be completely assessed.

Adequate sampling of the tumour-myometrial interface is necessary to evaluate the degree of infiltration of the tumour into the myometrium, correctly classify the tumour and thus properly treat the patient.

\section{DISTINCTION OF ESN FROM LOW-GRADE ESS WITH LIMITED INFILTRATION}

These two lesions are grossly indistinguishable, as they have well-defined pushing borders in relationship to the surrounding myometrium. ${ }^{11}$ On microscopic examination, low-grade ESS with limited infiltration is defined as a tumour that does not fulfil the criteria for an ESN (having $<3$ tongues or nodules at the most $3 \mathrm{~mm}$ in largest dimension) and does not have the overt permeative growth of a low-grade ESS or the associated vascular invasion (fig 5). These tumours have been recently described in a series of 50 ESNs that included three such tumours. ${ }^{11}$ Follow-up was available for only one patient with a tumour that had six tongues or detached nodules from the main mass ranging from 1 to $5 \mathrm{~mm}$, but it was limited to 62 months. The tumour was extensively sampled, with 51 slides showing the tumour-myometrium interface. The behaviour of these tumours

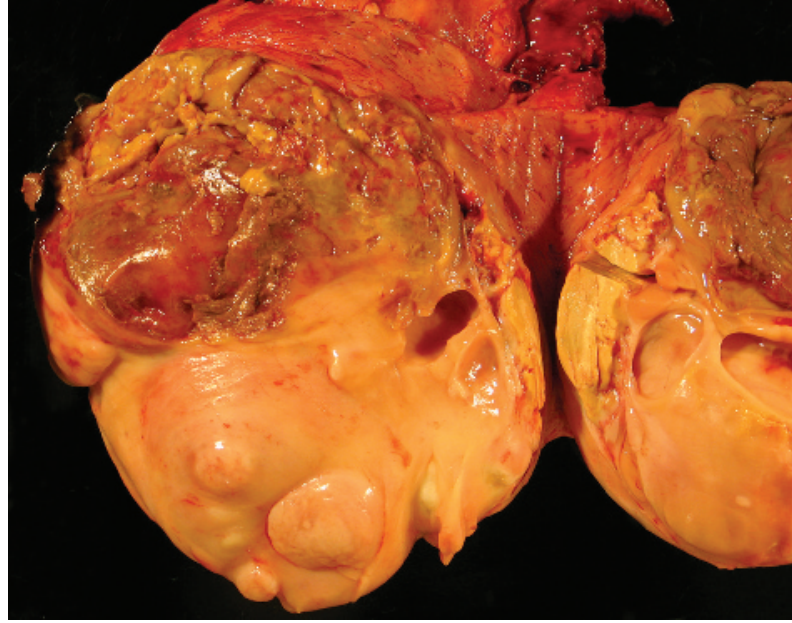

Figure 2 Endometrial stromal nodule. A large well-circumscribed mass shows a tan cut surface, scattered cysts and an extensive area of infarction.

is very difficult to predict, as studies with long follow-up are scarce. Currently, these tumours are best diagnosed as low-grade ESSs with an explanatory note stating that the tumour is not as overtly invasive as a typical low-grade ESS, and for this reason the tumour may behave in a more benign fashion.

\section{Potential pitfall}

Relying on the gross circumscription may lead to undersampling of the tumour and therefore to a diagnosis of ESN.

\section{Recommendations}

The tumor-myometrium interface should be sampled to identify invasive foci.

For practical purposes and owing to limited experience with these tumours, it is currently best to report them as low-grade ESS, with an explanatory note describing that the degree of invasion is much less than that seen in conventional low-grade ESS.

\section{DISTINCTION OF LOW-GRADE ESS FROM HIGH- GRADE ESS AND UES}

On gross examination, high-grade ESS and UES show a destructive infiltrative growth into the myometrium, which

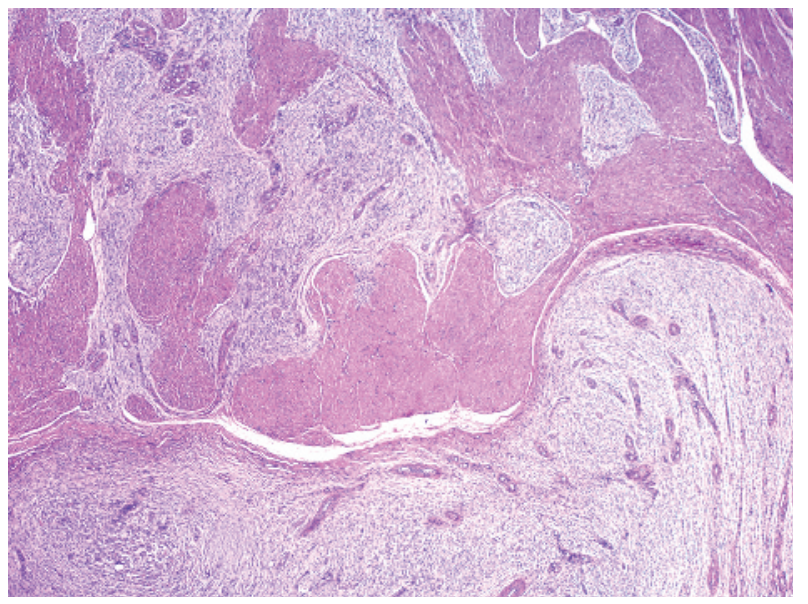

Figure 3 Low-grade endometrial stromal sarcoma. Irregular nests and islands of blue cells with a prominent delicate vascular network diffusely infiltrate the myometrium. 


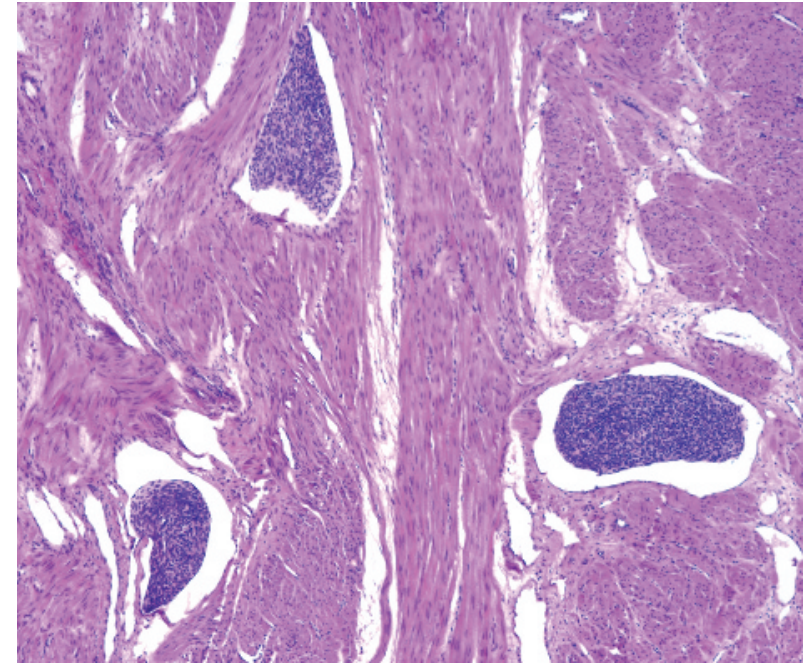

Figure 4 Low-grade endometrial stromal sarcoma. Nests of neoplastic endometrial stromal cells are present in vascular spaces.

contrasts with the permeative invasion of the myometrium and myometrial vessels seen in low-grade ESS. ${ }^{256}$ Both tumours have a grey, fleshy, cut surface often associated with areas of necrosis. Microscopically, marked degrees of nuclear atypia are absent in low-grade ESS, but are characteristic of high-grade ESS and UES. Although low-grade EES often shows mitotic rates $<3$ in 10 high-power fields, higher rates do not exclude this diagnosis, and it is now accepted that mitotic activity is not important in classifying an ESS as low or high grade. ${ }^{56}$ If a tumour has the typical morphology of an EST with the characteristic tongue-like growth into the myometrium and/or myometrial veins, it should be classified as a low-grade ESS regardless of the mitotic counts, as mitotic counts do not influence prognosis in these tumours. The most important feature for distinguishing low-grade from high-grade tumours is the resemblance of the neoplastic cells to proliferative endometrial stroma. The diagnosis of high-grade ESS should be made only in cases where a component of low-grade ESS may be recognised; otherwise, the diagnosis is that of UES. ${ }^{3}$ It is extremely important to distinguish low-grade from high-grade tumours, as the high-grade tumours behave as high-grade sarcomas and carry a poor prognosis. ${ }^{5}$ UESs should be diagnosed only after extensive sampling has excluded smooth or skeletal muscle differentiation, as this would result in a diagnosis of high-grade leiomyosarcoma or rhabdomyosarcoma. Small foci of carcinoma admixed with the sarcomatous component would favour a malignant mixed mullerian tumour, whereas the finding of typical adenosarcoma would clinch the diagnosis of adenosarcoma with sarcomatous overgrowth. This may be difficult in curettage specimens, owing to the limited representation of the tumour. CD10 expression is not helpful in this differentiation as high-grade ESSs as well as leiomyosarcomas, rhabdomyosarcomas and malignant mixed mullerian tumours, express this marker. ${ }^{23-25}$ The differential diagnosis is largely based on morphology. Smooth-muscle markers and myogenin or myoDl may be used to rule out a leiomyosarcoma or rhabdomyosarcoma respectively, or to identify a rhabdomyosarcoma component in a malignant mixed mullerian tumour.

\section{Potential pitfall}

A high mitotic rate may lead to a misdiagnosis of high-grade ESS.

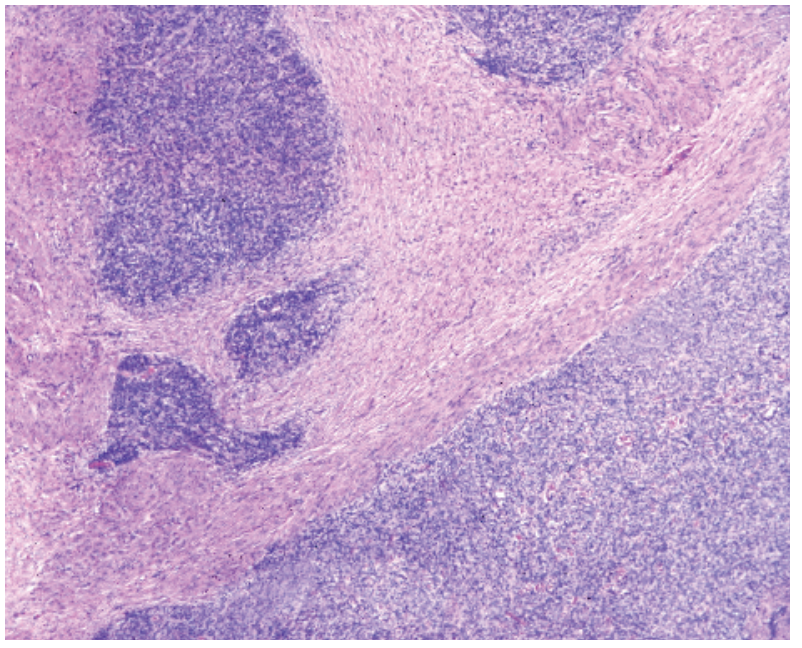

Figure 5 Endometrial stromal sarcoma with limited infiltration. Several distinct islands of the tumour, some showing a typical tongue-like configuration, are seen in close proximity to the main mass. Occasional nests are $>3 \mathrm{~mm}$ away.

\section{Recommendations}

If the histological features of the tumour are reminiscent of endometrial stroma the diagnosis should be low-grade ESS. The diagnosis of high-grade ESS can be made only when a component of low-grade ESS is recognised and, therefore, UES is a diagnosis of exclusion. Extensive sampling of the tumour is necessary to rule out other high-grade uterine tumours.

\section{DISTINCTION OF EST FROM HIGHLY CELLULAR LEIOMYOMA}

The clinical presentation of highly cellular leimyomas (HCLs) is non-specific, as occurs with most leiomyomas. However, these tumours may be confused with ESTs on gross and microscopic examination. ${ }^{26}$ On gross examination, HCLs have an appearance that closely overlaps with that seen in ESTs as they are yellow or yellow-tan and have a soft consistency (fig 6), in contrast with conventional leiomyomas, which are typically white with a firm cut surface. On histological examination, shared features include hypercellularity and prominent vascularity (fig 7A), as well as, in some cases, the finding of an irregular margin in relation to the surrounding myometrium. Helpful histological clues in this differential diagnosis are as follows:

- Focal merging of the highly cellular areas with typical fascicular areas of smooth-muscle neoplasia, more commonly seen at the periphery of the tumour in most cases.

- Large thick muscular-walled blood vessels throughout the tumour, in contrast with the arterioles of an endometrial stromal neoplasm (fig 7A). On occasion, some large, thickwalled blood vessels may be found at the junction of the EST and the myometrium; however, these are most likely entrapped.

- Cleft-like spaces, some apparently representing compressed vessels, others apparently the result of oedema. ${ }^{26}{ }^{27}$

In cases where the diagnosis is difficult to establish by light microscopy, immunohistochemical analysis may be helpful in arriving at the correct diagnosis, which is crucial owing to differences in treatment and prognosis. ${ }^{26}$ If the diagnosis of HCL is based on curettage material, the tumour can be managed conservatively, whereas given the diagnosis of an 


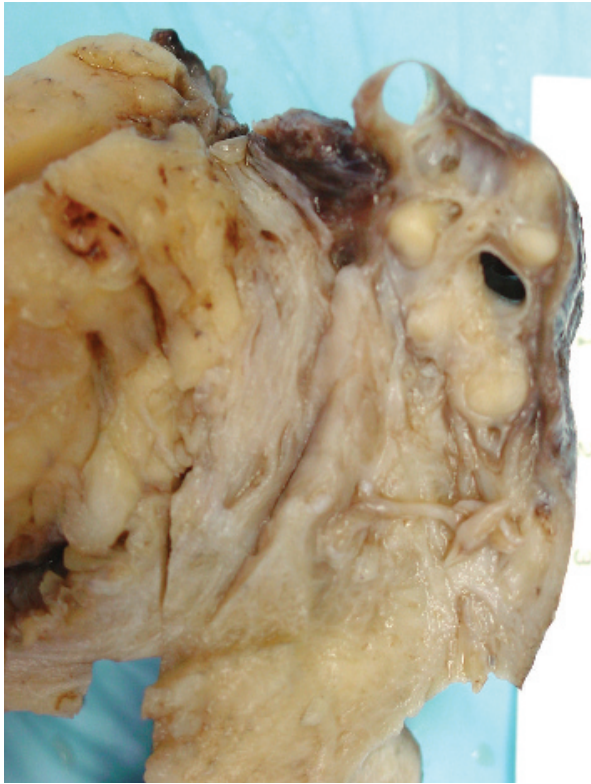

Figure 6 Highly cellular leiomyoma associated with intravenous leiomyomatosis. A tan to yellow mass with a slightly irregular margin is associated with tan worm-like plugs of tumour distending vascular spaces.

EST, the patient will need hysterectomy to further categorise the tumour.

The neoplastic endometrial stromal cells are typically immunoreactive for vimentin, muscle-specific and smoothmuscle actin, and may be positive for keratin and desmin..$^{28-33}$ Thus, this panel of antibodies is not helpful in distinguishing EST from HCL or from leiomyosarcoma, the two most common tumours in the differential diagnosis with EST.

Although CD10 was initially thought to be a specific marker of ESTs, ${ }^{34}{ }^{35}$ it has been shown that CD10 is expressed in smooth-muscle tumours of the uterus, most commonly in leiomyosarcomas and HCLs (fig 7B). ${ }^{33}$ Other antibodies useful in this differential diagnosis include h-caldesmon (fig 7C), ${ }^{24}{ }^{33}{ }^{37-39}$ histone deacetylase $8,^{40}$ and smooth-muscle myosin. ${ }^{40}{ }^{41}$ However, it is important to remember that ESTs may have areas with smooth-muscle differentiation that express these markers, underscoring the importance of correlating immunohistochemical results with the morphological findings. ${ }^{2434}$ Furthermore, leiomyosarcomas may be hcaldesmon negative, ${ }^{38}$ indicating that a panel of antibodies rather than a single antibody should be used in the differential diagnosis of ESTs and smooth-muscle tumours. Finally, oxytocin receptor, a neurohypophysial peptide associated with muscle contraction during labour, stains all conventional leimyomas and HCLs as well as leiomyosarcomas but is not expressed in ESTs ${ }^{24}$; however, this antibody is not widely used in daily practice at present.

\section{Potential pitfalls}

The finding of prominent cellularity (small blue-cell tumour) in the uterus may lead to the wrong diagnosis of EST, especially as the margin with the surrounding myometrium may appear sharper and worrisome for the "tongue-like" growth of a lowgrade ESS. The use of a single antibody, especially CD10, may lead to a wrong final diagnosis.

\section{Recommendations}

Fascicular areas, thick-walled blood vessels and cleft-like spaces in the tumour, more often seen at the periphery of the tumour, and the transition of the tumour cells with the surrounding myometrium should be looked for. A panel of antibodies that includes CDI0 and two muscle markers should be used. The immunohistochemical findings should be correlated with the morphological findings.

\section{DISTINCTION OF LOW-GRADE ESS FROM HIGHLY CELLULAR INTRAVENOUS LEIOMYOMATOSIS}

The clinical presentation of intravenous leiomyomatosis (IVL) is non-specific; however, as with low-grade ESS, this lesion is present outside the uterus at the time of diagnosis in at least $30 \%$ of patients. In some, extension of the tumour into the inferior vena cava and the right side of the heart may result in cardiac manifestations, a feature rarely seen in low-grade ESS. ${ }^{42} 43$

On gross examination, cellular IVL may be misinterpreted as an ESS, because of its prominent intravascular growth, a soft tan to yellow cut surface and frequent association with a
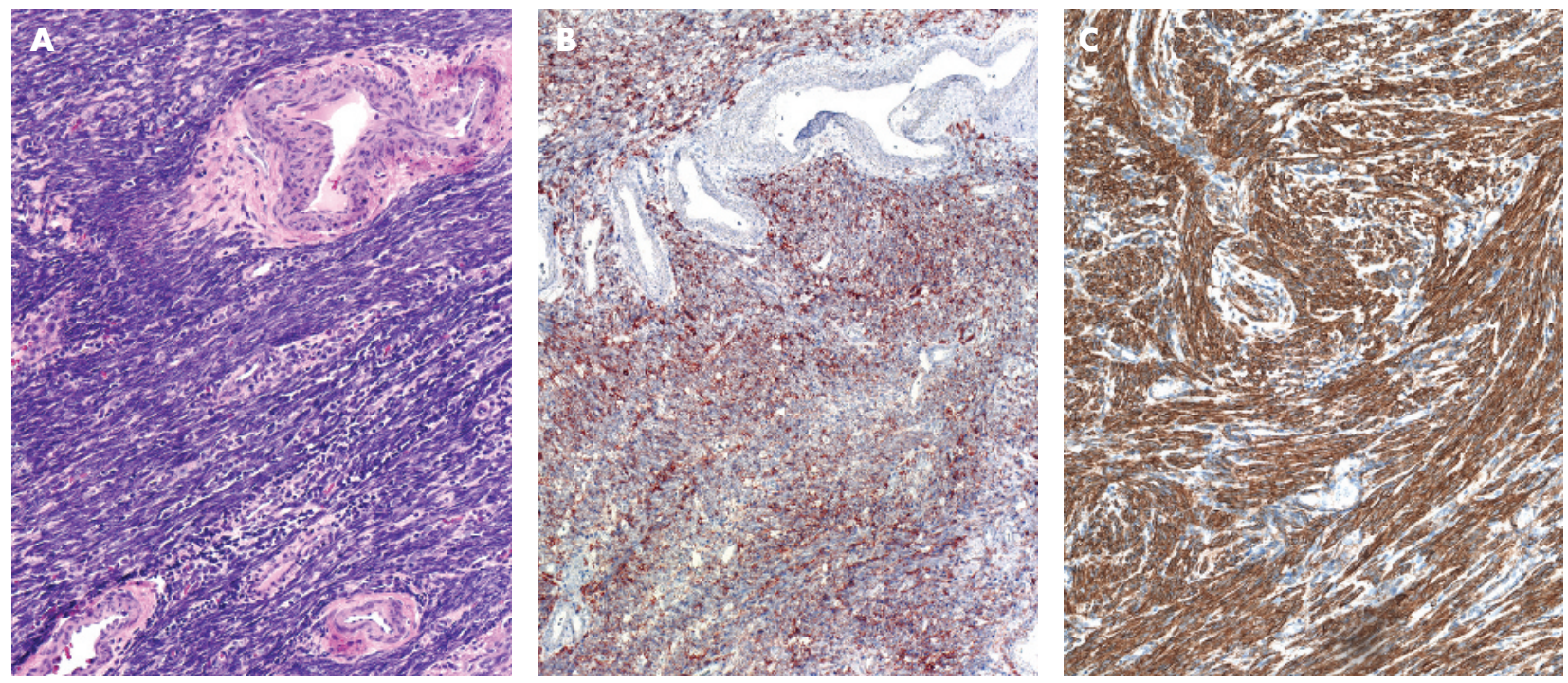

Figure 7 Highly cellular leiomyoma. A densely cellular proliferation of small spindle cells with elongated nuclei and scant cytoplasm is associated with large thick-walled vessels (A). The smooth-muscle cells are positive for CD10 (B) and for h-caldesmon (C). 


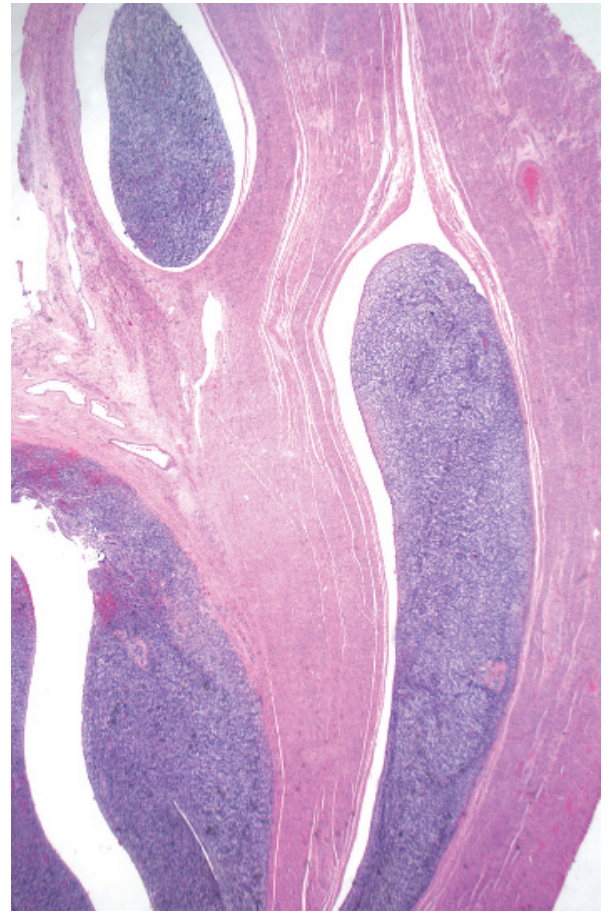

Figure 8 Intravenous leiomyomatosis. Prominent intravascular growth of small blue cells mimics the growth of endometrial stromal sarcoma.

conventional leiomyoma that may be confused with the main mass of an ESS (fig 8). ${ }^{44} 45$ Microscopically, the dense cellularity resembling a HCL may increase confusion with a low-grade ESS (fig 9). Helpful morphological features include all those typically seen in conventional leiomyomas, including the following:

- clefted or lobulated contour of the intravascular masses,

- focal fascicular architecture,

- cells with blunt-ended nuclei,

- prominent thick-walled vessels and

- hydropic change. ${ }^{44}$

Finally, other clues to the diagnosis of IVL include low mitotic activity in most tumours and the presence of tumour growth beneath the vascular endothelium with colonisation of the walls of veins. However, this phenomenon is seen in only a minority of cases. ${ }^{46}$

Low-grade ESS and IVL may recur months to years after the initial treatment because of growth of residual tumour in vascular spaces. ${ }^{42}$ It is, however, important to differentiate between those tumours, as about $50 \%$ of patients with lowgrade ESS develop pelvic recurrences, a pattern of spread not seen in IVL, ${ }^{6} 151644$ and also because the main treatment for IVL is still surgical resection, whereas patients with low-grade ESS often receive adjuvant radiation or hormonal treatment. ${ }^{122} 42$

\section{Potential pitfall}

Intravascular growth pattern of small blue cells may first be considered a low-grade ESS

\section{Recommendations}

Fascicular areas, thick-walled blood vessels, cleft-like spaces, as seen in HCLs, and tumour growth beneath the vascular endothelium should be looked for. A panel of antibodies should be used that includes CD10 and two muscle markers.
The immunohistochemical findings should be correlated with the morphological findings.

\section{DISTINCTION OF EST FROM PERIVASCULAR EPITHELIOD CELL TUMOUR}

The perivascular epithelioid cell tumour (PEComa) is a newly described low-grade mesenchymal tumour. It belongs to the family of lesions that includes the clear-cell "sugar" tumours of the lung and pancreas, some forms of angiomyolipoma and rare tumours in other locations. ${ }^{47}$ All these tumours originate from the perivascular epithelioid cell, which is a cell defined by the presence of abundant clear to eosinophilic granular cytoplasm and positive staining for human melanoma black 45 , as well as frequent expression of muscle markers. Interestingly, some tumours are associated with lymphangiomyomatosis as well as tuberous sclerosis, a feature not described in ESTs. ${ }^{47}$

On gross examination, PEComas may show poorly defined margins, with a fleshy, soft, cut surface that ranges from greywhite to tan or yellow, resembling the appearance of an EST. ${ }^{48}$

On examination by low-power microscopy, some tumours have a tongue-like infiltrative growth resembling the infiltrative pattern seen in low-grade ESS. ${ }^{48}$ The tumour cells have abundant clear to eosinophilic cytoplasm that may grow in sheets, with a scant amount of intervening stroma and a prominent network of small blood vessels (fig 10). ${ }^{47-50}$ Most of the aforementioned features, including eosinophilic or clear cells with abundant cytoplasm, may be seen in ESTs. ${ }^{51} 52$ However, PEComas show a predominantly nested growth, which is often associated with a focal fascicular growth of cells with elongated nuclei having a similar appearance to that seen in smooth-muscle tumours. Finally, the cells tend to be arranged in a radial fashion around the vessels. ${ }^{47}$ These features are not encountered in ESTs, and, furthermore, ESTs will show at least focally some areas that resemble the normal endometrial stroma with arterioles.

On immunohistochemical studies, ESTs may be positive for smooth-muscle markers and PEComas may be positive for $\mathrm{CDIO}^{23} 4748$; thus, these markers are not helpful in this differential diagnosis. However, in contrast with PEComas, ESTs do not express human melanoma black 45, Melan-A or microphthalmia factor. ${ }^{47} 48$

\section{Potential pitfall}

CD10 can be positive in PEComa and it may thus be erroneously diagnosed as EST.

\section{Recommendation}

When studying a tumour with clear cells, PEComa should always be considered in the differential diagnosis. A panel of antibodies should be used when considering more than one tumour in the differential diagnosis, as tumours may show overlapping results with isolated antibodies.

\section{EST VARIANTS}

ESTs may show different types of differentiation including smooth muscle, ${ }^{53-55}$ fibrous or myxoid change, ${ }^{55-57}$ sex cord-like elements, ${ }^{11}{ }^{58-61}$ glandular differentiation, ${ }^{62}{ }^{63}$ epithelioid, ${ }^{52}$ rhabdoid $^{6164-66}$ or clear cells, ${ }^{51}$ rhabdomyoblastic differentiation, ${ }^{6768}$ and granular change. ${ }^{52}$ Fatty metaplasia and bizarre nuclei have recently been reported in these tumours ${ }^{68}$ In this category, the most common problems in differential diagnosis-namely, smooth-muscle and sex cord-like differentiation-will be discussed

EST with smooth muscle differentiation versus smoothmuscle tumour

On gross examination, endometrial stromal tumours with smooth-muscle differentiation (ESTs-SMD) often show an 


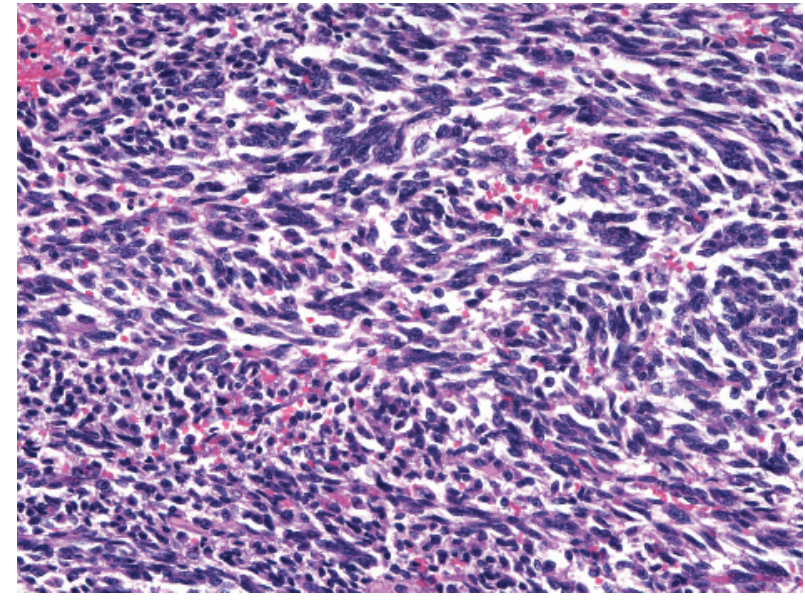

Figure 9 Intravascular leiomyomatosis. Loosely spindle-shaped cells growing inside vascular spaces are seen. The intravascular growth is associated with a highly cellular leiomyoma (not shown).

admixture of soft tan to yellow nodules and firm white whorled nodules, or alternatively the paler, firmer tissue may be seen at the periphery of softer nodules, a picture that is not characteristic of smooth muscle tumours in general (figs 11, 12). To establish the diagnosis of mixed EST-SMD, the smoothmuscle component should occupy at least $30 \%$ of the neoplasm as seen by haematoxylin and eosin staining. ${ }^{27}$ In many cases, the smooth-muscle component characteristically shows nodules with central hyalinisation (starburst pattern), which merge with disorganised short fascicles or long mature fascicles of the smooth muscle, a feature almost never encountered in conventional smooth-muscle tumours (fig 13). ${ }^{53-55}$ Furthermore, conventional areas of endometrial stromal neoplasia are present in ESTs-SMD, confirming the endometrial stromal origin of the tumour.

Immunohistochemical staining should be interpreted with caution in these cases, as the smooth-muscle component is often positive for CD10 and smooth-muscle markers and this profile may be considered to be diagnostic of smooth muscle neoplasia. ${ }^{24}{ }^{33}$ Immunohistochemical staining should be correlated with the different morphological components of the tumour. Conventional areas of endometrial stromal neoplasia should be positive for CDl0 but not positive for $>1$ smoothmuscle marker, being more often positive for muscle actin and desmin.

\section{Potential pitfalls}

If the smooth muscle component, particularly when it is mature and relatively well organised, is misconstrued as myometrium, a well-circumscribed tumour may be misinterpreted as an invasive EST and hence an ESS. It is crucial to appreciate that in such cases, we are examining regions with divergent differentiation in the mass itself rather than myometrial invasion by a low-grade ESS.

Coexpression of CDI0 and smooth-muscle markers in areas of SMD may lead to the diagnosis of a smooth-muscle tumour.

\section{Recommendations}

The term "stromomyoma" should not be used because it implies that the tumour is an ESN. These tumours should be reported as ESNs or ESSs with SMD (depending on the margins), with the designation "mixed endometrial stromalsmooth-muscle tumour" given in parentheses, as the margin is the most important prognostic parameter.

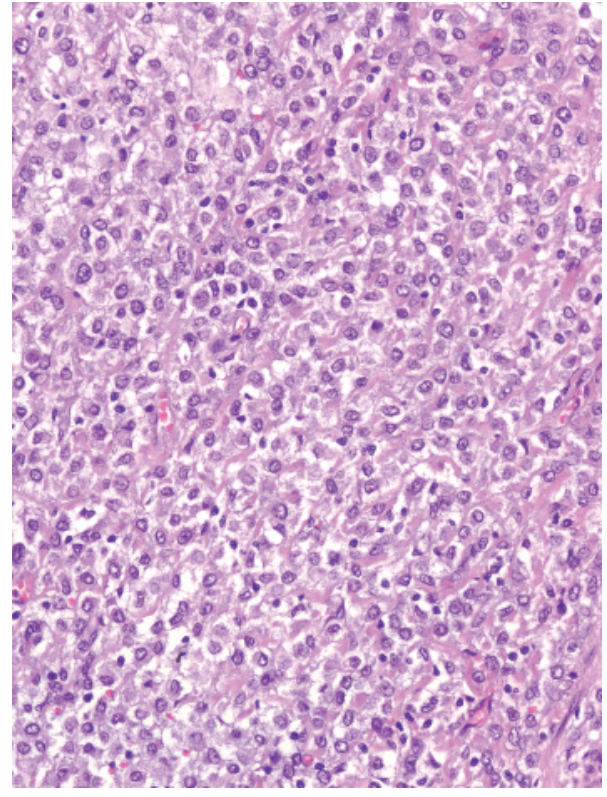

Figure 10 Perivascular epitheliod cell tumour is composed of sheets of cells with abundant clear cytoplasm.

The results by immunohistochemistry should be interpreted in correlation with the appearance of the different areas identified on histological examination.

\section{EST WITH SEX CORD-LIKE DIFFERENTIATION VERSUS UTERINE TUMOUR RESEMBLING AN OVARIAN SEX CORD STROMAL TUMOUR}

Uterine tumour resembling an ovarian sex cord stromal tumour (UTROSCT) is strictly defined as a tumour with prominent sex cord-like differentiation in which there is no conspicuous endometrial stromal background. ${ }^{58}$ The age and clinical presentation is similar for patients with UTROSCT and for those with ESTs except for the fact that the patients with UTROSCT almost never present with metastases.

On gross examination, UTROSCT typically appears as a circumscribed myometrial or submucosal mass that is soft and ranges from grey to tan to yellow, an appearance that overlaps with that of an ESN.

On microscopic examination, ESN and low-grade ESS may show sex cord-like differentiation (fig 14), and the histological

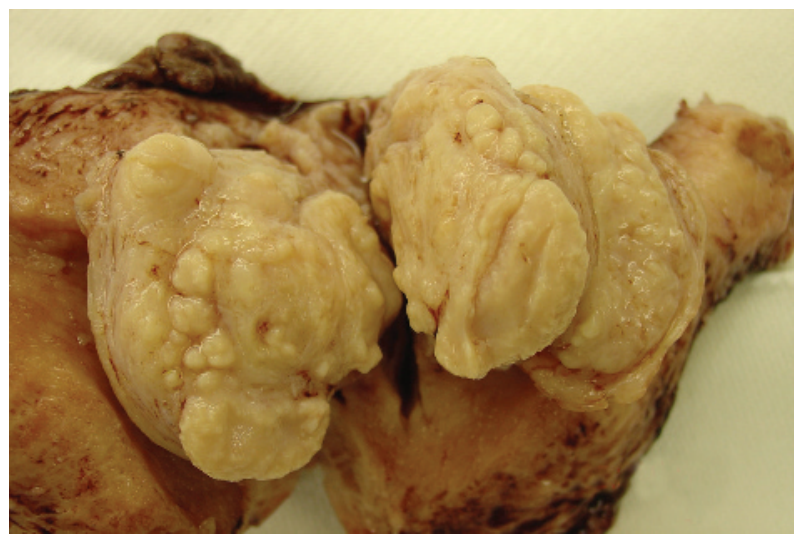

Figure 11 Mixed endometrial stromal and smooth-muscle tumour. A wellcircumscribed multilobular mass protrudes from the myometrium. It shows firm pale nodules alternating with irregular tan areas. 


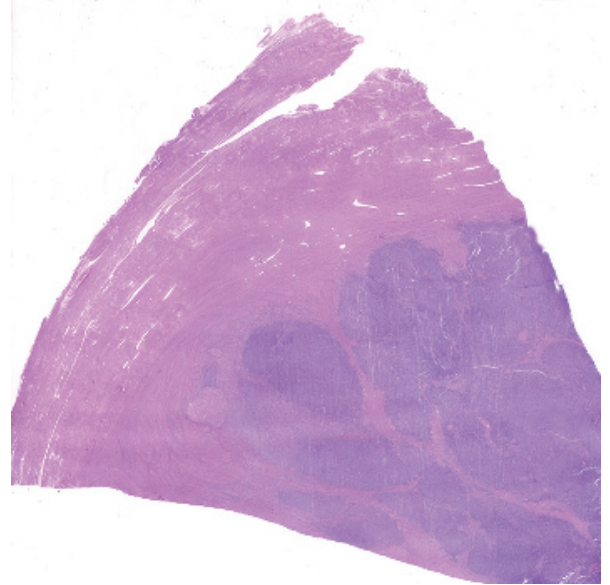

Figure 12 Mixed endometrial stromal and smooth-muscle tumour. A wellcircumscribed tumour composed of smooth muscle and endometrial stroma is abutting the surrounding myometrium. The smooth-muscle component forms a discrete pink rim at the periphery and coarse bundles that alternate with the endometrial stromal component.

appearance of some UTROSCTs may merge imperceptibly with that of ESTs when exhibiting a less than predominant sex cordlike pattern. ${ }^{115859}$ The histological patterns that create a resemblance to those of ovarian sex cord tumours, especially granulosa-cell and Sertoli-cell tumours, alone or in combination, should be the only elements present in the tumour to establish the diagnosis of UTROSCT. ${ }^{585969}$

Immunohistochemical analysis may be of help in this differential diagnosis. Inhibin, the most specific marker for sex cord stromal tumours of the ovary, is typically negative in pure ESTs, ${ }^{33} 59$ 70-72 but it is often positive in areas of sex cordlike differentiation in ESTs and in UTROSCTs (fig 15A), although positivity varies in intensity and percentage. ${ }^{73}$ Calretinin and CD99 may also stain normal sex cord elements as well as UTROSCTs (fig 15B), but they are negative in conventional areas of EST. ${ }^{59-74}$ Melan A may show positive staining in sex cord-like cells, consistent with the presence of steroid-producing cells, and is supportive of their specialised gonadal stromal nature, but it is negative in pure endometrial stromal areas..$^{59}{ }^{73}$ Finally, CD10, typically positive in ESTs, has been recently reported to be positive also in UTROSCTs. ${ }^{59}$ These results underscore once again the importance of correlating the immunohistochemical findings with the diverse morphological areas of a given tumour, indicating in this particular scenario that positivity for any of these markers does not establish unequivocally a diagnosis of UTROSCT.

Finally, it is important to differentiate UTROSCT from lowgrade ESS, as UTROSCT typically behaves in a benign fashion, ${ }^{69} 75$ whereas patients with low-grade ESSs have frequent recurrences. ${ }^{67162122}$

\section{Potential pitfalls}

Fragments of tumour seen in a curettage specimen that are predominantly composed of sex cord-like elements may lead to the automatic diagnosis of UTROSCT, without consideration of an EST with sex cord-like differentiation.

Positivity for any marker of sex cord differentiation may erroneously lead to a diagnosis of UTROSCT.

\section{Recommendations}

It is necessary to sample the tumour extensively to rule out an endometrial stromal component and thus be able to classify the tumour as a UTROSCT.

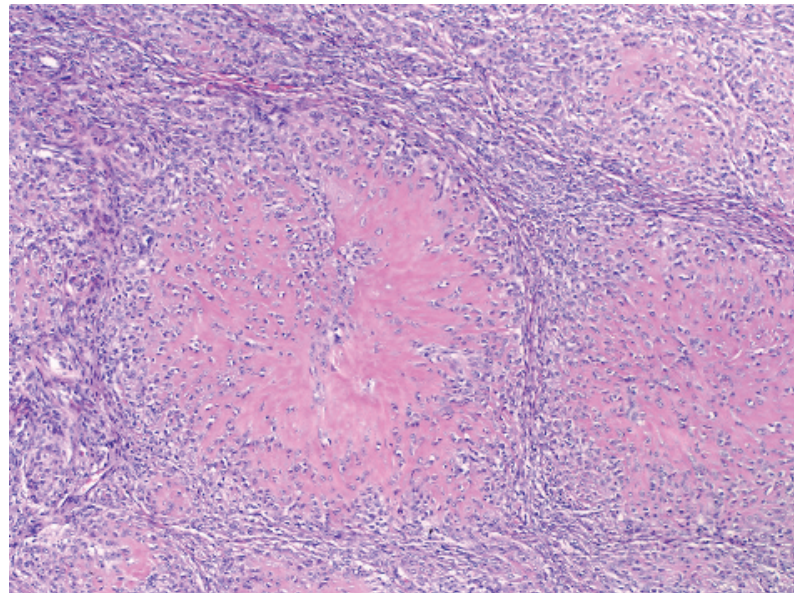

Figure 13 Mixed endometrial stromal and smooth-muscle tumour. A central area of hyalinisation shows radiating fibres of collagen that entrap plump neoplastic cells (starburst pattern), which in turn merge with immature bundles of smooth muscle.

The distinction of an EST with sex cord differentiation from a UTROSCT can be made only in a hysterectomy specimen. It is important to correlate the immunohistochemical findings with the diverse morphological areas of a given tumour, as areas without sex cord differentiation in an EST do not stain for most markers listed above.

\section{NEW DEVELOPMENTS}

It is well known that ESTs often contain oestrogen and progesterone receptors, findings that may have therapeutic and prognostic implications. ${ }^{76}{ }^{77}$ However, the presence of these receptors has limited utility in differential diagnosis in as much as these receptors may be found in many other epithelial and mesenchymal tumours of the uterus. Aromatase participates in extraovarian oestrogen production via conversion of androgen to oestrogen through the aromatase enzyme complex. ${ }^{78}$ Aromatase has been detected in stromal cells of endometriosis, adenomyosis and endometrial carcinomas, as well as ESSs, and in the latter no expression of aromatase tended to correlate with stage I disease in one study. ${ }^{78}$ The importance of that finding is the introduction of aromatase inhibitors as a new modality of treatment for low-grade ESSs. ${ }^{17-20}$

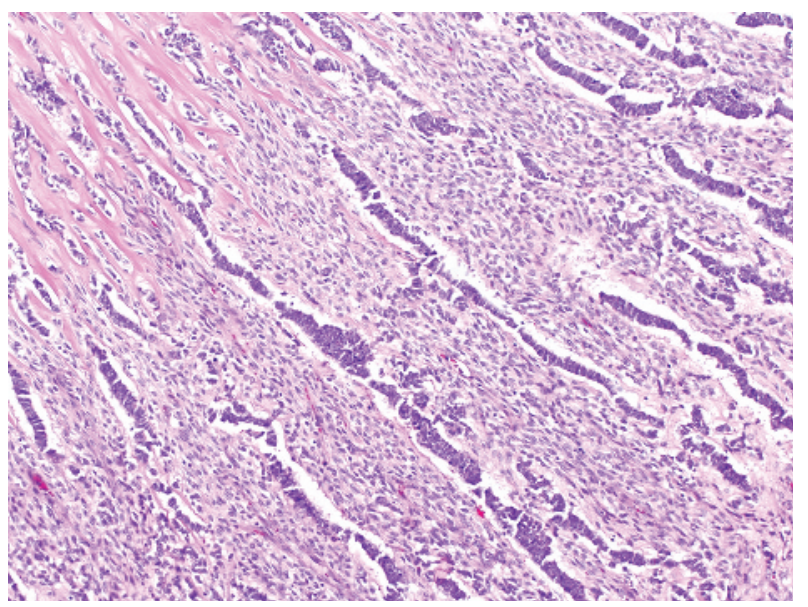

Figure 14 Low-grade endometrial stromal tumour with epithelial-like differentiation. Elongated cords are present in a background of endometrial stromal neoplasia. 

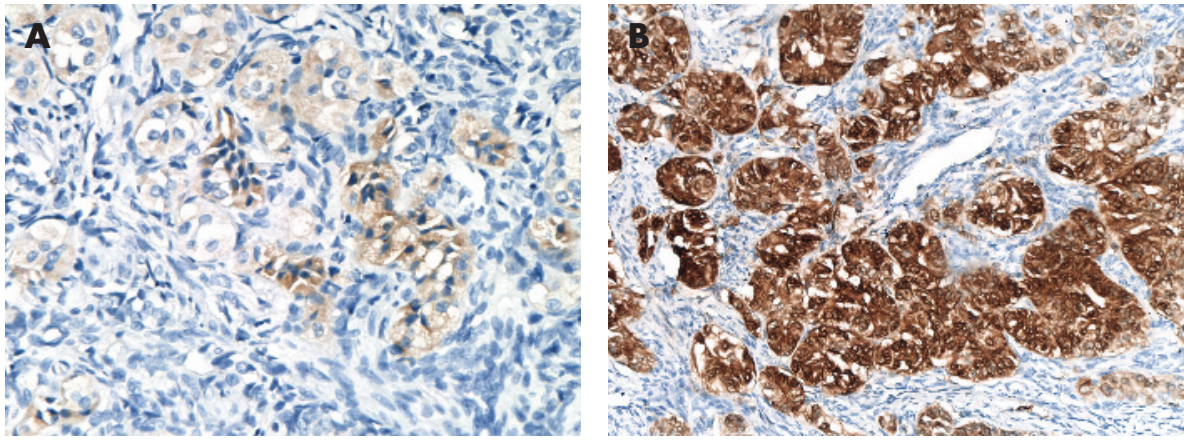

Figure 15 A uterine tumour resembling an ovarian sex cord stromal tumour shows (A) inhibin and (B) calretinin positivity.

Although initial studies on c-kit in ESTs detected absence of staining, ${ }^{33}$ recent studies have shown some c-kit expression in ESTs. ${ }^{79-81}$ However, in contrast with gastrointestinal stromal tumours where this expression can be used for treatment purposes, the experience with ESTs is limited and contradictory.

Genetic studies have shown that $\mathrm{t}(7 ; 17)$ is the most common translocation encountered in conventional ESTs, ${ }^{82}$ with involvement of two zinc finger genes, JAZFl and JJAZl, ${ }^{83}$ in conventional ESTs as well as in ESTs-SMD and in those with fibrous or myxoid change. ${ }^{85} 86$ Other genetic alterations, most often loss of heterozygosity of phosphatase and tensin homolog deleted on chromosome 10, have also been reported in ESTs. ${ }^{1}$ However, these findings do not at the moment have any prognostic or therapeutic implications.

\section{Authors' affiliations}

Patricia Baker, Pathology Department, Health Sciences Centre, Winnipeg, Manitoba, Canada

Esther Oliva, Pathology Department, Massachusetts General Hospital, Boston, Massachusetts, USA

\section{Take-home messages}

- In most instances, the diagnosis of endometrial stromal tumour (EST) may be established on morphology alone.

- Extensive sampling may be important in distinguishing endometrial stromal neoplasia from an endometrial stromal sarcoma (ESS).

- A tumour resembling endometrial stroma should be classified as a low-grade ESS and not as a high-grade ESS.

- Awareness of the existence of EST variants will help in the correct classification of the tumour.

- CD10 positivity in a uterine tumour is not diagnostic of EST.

- A panel of antibodies rather than a single antibody should be used when interpreting mesenchymal tumours of the uterus.

- The most useful panel includes CD10 and two smooth muscle markers.

- Diffuse or multifocal and strong positivity for two muscle markers favours a smooth-muscle nature of the tumour despite CD10 positivity.

- Immunohistochemical results should always be correlated with the histological appearance of the fumour and interpreted accordingly, as ESTs may show areas with different types of differentiation.
Competing interests: None declared.

\section{REFERENCES}

1 Moinfar F, Kremser ML, Man YG, et al. Allelic imbalances in endometrial stromal neoplasms: frequent genetic alterations in the nontumorous normalappearing endometrial and myometrial tissues. Gynecol Oncol 2004:95:662-71

2 Hendrickson MR, Tavassoli FA, Kempson RL, et al. Mesenchymal tumors and related lesions. Pathology and genetics of tumours of the breast and female organs. Lyon: IARC Press, 2003:233-6.

3 Oliva E, Clement PB, Young RH. Endometrial stromal tumors: an update on a group of tumors with a protean phenotype. Adv Anat Pathol 2000;7:257-81

4 Oliva E, Clement PB, Young RH. Mesenchymal tumours of the uterus: selected topics emphasizing diagnostic piffalls. Curr Diagn Pathol 2002;8:268-82.

5 Evans $\mathrm{HL}$. Endometrial stromal sarcoma and poorly differentiated endometrial sarcoma. Cancer 1982;50:2170-82.

6 Chang KL, Crabtree GS, Lim-Tan SK, et al. Primary uterine endometrial stromal neoplasms. A clinicopathologic study of 117 cases. Am J Surg Pathol 1990:14:415-38.

7 Fekete PS, Vellios F. The clinical and histologic spectrum of endometrial stromal neoplasms: a report of 41 cases. Int J Gynecol Pathol 1984;3:198-212.

8 Young RH, Prat J, Scully RE. Endometrioid stromal sarcomas of the ovary. A clinicopathologic analysis of 23 cases. Cancer 1984;53:1143-55.

9 Young RH, Scully RE. Sarcomas metastatic to the ovary: a report of 21 cases. Int J Gynecol Pathol 1990;9:231-52.

10 Tavassoli FA Norris HJ. Mesenchymal tumours of the uterus. VII. A clinicopathological study of 60 endometrial stromal nodules. Histopathology 1981;5:1-10

11 Dionigi A, Oliva E, Clement PB, et al. Endometrial stromal nodules and endometrial stromal tumors with limited infiltration: a clinicopathologic analysis of 50 cases. Am J Surg Pathol 2002:26:567-81.

12 Hart WR, Yoonessi M. Endometrial stromatosis of the uterus. Obstet Gynecol 1977;49:393-403

13 Vilos GA, Harding PG, Sugimoto AK, et al. Hysteroscopic endomyometrial resection of three uterine sarcomas. J Am Assoc Gynecol Laparosc $2001 ; 8: 545-51$.

14 Schilder JM, Hurd WW, Roth LM, et al. Hormonal treatment of an endometrial stromal nodule followed by local excision. Obstet Gynecol 1999;93:805-7.

15 Bodner K, Bodner-Adler B, Obermair A, et al. Prognostic parameters in endometrial stromal sarcoma: a clinicopathologic study in 31 patients. Gynecol Oncol 2001;81:160-5.

16 De Fusco PA, Gaffey TA, Malkasian GD Jr, et al. Endometrial stromal sarcoma: review of Mayo Clinic experience, 1945-1980. Gynecol Oncol 1989:35:8-14.

17 Maluf FC, Sabbatini P, Schwartz L, et al. Endometrial stromal sarcoma: objective response to letrozole. Gynecol Oncol 2001;82:384-8

18 Spano JP, Soria JC, Kambouchner M, et al. Long-term survival of patients given hormonal therapy for metastatic endometrial stromal sarcoma. Med Oncol 2003;20:87-93.

19 Leunen M, Breugelmans $M$, De Sutter $P$, et al. Low-grade endometrial stromal sarcoma treated with the aromatase inhibitor letrozole. Gynecol Oncol 2004:95:769-71.

20 Leiser AL, Hamid AM, Blanchard R. Recurrence of prolactin-producing endometrial stromal sarcoma with sex-cord stromal component treated with progestin and aromatase inhibitor. Gynecol Oncol 2004;94:567-71.

21 Piver MS, Rutledge FN, Copeland L, et al. Uterine endolymphatic stromal myosis: a collaborative study. Obstet Gynecol 1984;64:173-8.

22 Gloor E, Schnyder P, Cikes M, et al. Endolymphatic stromal myosis. Surgical and hormonal treatment of extensive abdominal recurrence 20 years after hysterectomy. Cancer 1982;50:1888-93.

23 Oliva E. CD10 expression in the female genital tract: does it have useful diagnostic applications? Adv Anat Pathol 2004;11:310-15.

24 Loddenkemper C, Mechsner S, Foss HD, et al. Use of oxytocin receptor expression in distinguishing between uterine smooth muscle tumors and endometrial stromal sarcoma. Am J Surg Pathol 2003;27:1458-62. 
25 Mikami Y, Hata S, Kiyokawa T, et al. Expression of CD10 in malignant mullerian mixed tumors and adenosarcomas: an immunohistochemical study. Mod Pathol 2002; 15:923-30

26 Oliva E, Young RH, Clement PB, et al. Cellular benign mesenchymal tumors of the uterus. A comparative morphologic and immunohistochemical analysis of 33 highly cellular leiomyomas and six endometrial stromal nodules, two frequently confused tumors. Am J Surg Pathol 1995;19:757-68.

27 Clement PB. The pathology of uterine smooth muscle tumors and mixed endometrial stromal-smooth muscle tumors: a selective review with emphasis on recent advances. Int J Gynecol Pathol 2000; 19:39-55.

28 Abrams J, Talcott J, Corson JM. Pulmonary metastases in patients with low-grade endometrial stromal sarcoma. Clinicopathologic findings with immunohistochemical characterization. Am J Surg Pathol 1989;13:133-40.

29 Devaney K, Tavassoli FA. Immunohistochemistry as a diagnostic aid in the interpretation of unusual mesenchymal tumors of the uterus. Mod Pathol $1991 ; 4: 225-31$

30 Lillemoe TJ, Perrone T, Norris HJ, et al. Myogenous phenotype of epithelial-like areas in endometrial stromal sarcomas. Arch Pathol Lab Med $1991 ; 115: 215-19$.

31 Franquemont DW, Frierson HF, Mills SE. An immunohistochemical study of normal endometrial stroma and endometrial stromal neoplasms. Evidence for smooth muscle differentiation. Am J Surg Pathol 1991;15:861-70.

32 Farhood Al, Abrams J. Immunohistochemistry of endometrial stromal sarcoma. Hum Pathol 1991;22:224-30.

33 Oliva $E$, Young $\mathrm{RH}$, Amin $\mathrm{MB}$, et al. An immunohistochemical analysis of endometrial stromal and smooth muscle tumors of the uterus: a study of 54 cases emphasizing the crucial importance of using a panel because of overlap in immunoreactivity for individual antibodies. Am J Surg Pathol 2002;26:403-12.

34 Chu P, Arber DA. Paraffin-section detection of CD10 in 505 nonhematopoietic neoplasms. Frequent expression in renal cell carcinoma and endometrial stromal sarcoma. Am J Clin Pathol 2000;113:374-82.

35 Chu PG, Arber DA, Weiss LM, et al. Utility of CD10 in distinguishing between endometrial stromal sarcoma and uterine smooth muscle tumors: an immunohistochemical comparison of 34 cases. Mod Pathol 2001;14:465-71.

36 McCluggage WG, Sumathi VP, Maxwell P. CD10 is a sensitive and diagnostically useful immunohistochemical marker of normal endometrial stroma and of endometrial stromal neoplasms. Histopathology 2001;39:273-8.

37 Nucci MR, O'Connell JT, Huettner PC, et al. h-Caldesmon expression effectively distinguishes endometrial stromal tumors from uterine smooth muscle tumors. Am J Surg Pathol 2001;25:455-63.

38 Rush DS, Tan J, Baergen RN, et al. h-Caldesmon, a novel smooth muscle-specific antibody, distinguishes between cellular leiomyoma and endometrial stromal sarcoma. Am J Surg Pathol 2001;25:253-8.

39 Srodon M, Kurman RJ. CD10, desmin, and caldesmon in differentiating uterine stromal from smooth muscle tumors [abstract]. Mod Pathol 2002;15:211A.

40 De Leval L. Use of histone deacetylase 8 (HDAC8), a new marker of smooth muscle differentiation, in the classification of mesenchymal tumors of the uterus. Am J Surg Pathol 2006;30:319-27.

41 Malpica A, Averyt J, Deavers MT, et al. A comparison of the performance of smooth muscle myosin heavy chain (SMMS-1) versus caldesmon, desmin, and smooth muscle actin in the evaluation of uterine smooth muscle tumors [abstract 899]. Mod Pathol 2005; 18:194A.

42 Clement PB. Intravenous leiomyomatosis of the uterus. Pathol Annu 1988;23:153-83.

43 Tabata T, Takeshima N, Hirai Y, et al. Low-grade endometrial stromal sarcoma with cardiovascular involvement-a report of three cases. Gynecol Oncol 1999;75:495-8.

44 Clement PB, Young RH, Scully RE. Intravenous leiomyomatosis of the uterus. A clinicopathological analysis of 16 cases with unusual histologic features. Am J Surg Pathol 1988; 12:932-45.

45 Mulvany NJ, Slavin JL, Ostor AG, et al. Intravenous leiomyomatosis of the uterus: a clinicopathologic study of 22 cases. Int J Gynecol Pathol 1994;13:1-9.

46 Silverberg SG. Low-grade endometrial stromal sarcoma: a rare but often puzzling diagnostic problem. Pathol Case Rev 2000;5:173-80.

47 Folpe AL, Mentzel T, Lehr HA, et al. Perivascular epithelioid cell neoplasms of soft tissue and gynecologic origin: a clinicopathologic study of 26 cases and review of the literature. Am J Surg Pathol 2005;29:1558-75.

48 Vang R, Kempson RL. Perivascular epithelioid cell tumor ('PEComa') of the uterus: a subset of HMB-45-positive epithelioid mesenchymal neoplasms with an uncertain relationship to pure smooth muscle tumors. Am J Surg Pathol 2002;26:1-13.

49 Fukunaga M. Perivascular epithelioid cell tumor of the uterus: report of four cases. Int J Gynecol Pathol 2005;24:341-6.

50 Dimmler A, Seitz G, Hohenberger W, et al. Late pulmonary metastasis in uterine PEComa. J Clin Pathol 2003:56:627-8.

51 Lifschitz-Mercer B, Czernobilsky B, Dgani R, et al. Immunocytochemical study of an endometrial diffuse clear cell stromal sarcoma and other endometrial stromal sarcomas. Cancer 1987; 59: 1494-9.

52 Oliva E, Clement PB, Young RH. Epithelioid endometrial and endometrioid stromal tumors: a report of four cases emphasizing their distinction from epithelioid smooth muscle tumors and other oxyphilic uterine and extrauterine tumors. Int J Gynecol Pathol 2002;21:48-55.

53 Oliva E, Clement PB, Young RH, et al. Mixed endometrial stromal and smooth muscle tumors of the uterus: a clinicopathologic study of 15 cases. Am J Surg Pathol 1998;22:997-1005
54 Schammel DP, Silver SA, Tavassoli FA Combined endomerial stromal/smooth muscle neoplasms of the uterus. A clinicopathologic study of 38 cases [abstract] Mod Pathol 1999; 12:124A

55 Yilmaz A, Rush DS, Soslow RA. Endometrial stromal sarcomas with unusual histologic features: a report of 24 primary and metastatic tumors emphasizing fibroblastic and smooth muscle differentiation. Am J Surg Pathol 2002;26:1142-50.

56 Oliva E, Young RH, Clement PB, et al. Myxoid and fibrous variants of endometrial stromal tumors of the uterus: a report of 10 cases. Int J Gynecol Pathol 1999:18:310-19.

57 Kasashima S, Kobayashi M, Yamada M, et al. Myxoid endometrial stromal sarcoma of the uterus. Pathol Int 2003;53:637-41.

58 Clement PB, Scully RE. Uterine tumors resembling ovarian sex-cord tumors. A clinicopathologic analysis of fourteen cases. Am J Clin Pathol 1976:66:512-25.

59 Irving JA. Uterine tumors resembling ovarian sex cord tumors are polyphenotypic neoplasms with true sex cord differentiation. Mod Pathol 2006;19:17-24.

60 Zamecnik M, Michal M. Endometrial stromal nodule with retiform sex-cord-like differentiation. Pathol Res Pract 1998;194:449-53.

61 McCluggage WG, Date A, Bharucha $\mathrm{H}$, et al. Endometrial stromal sarcoma with sex cord-like areas and focal rhabdoid differentiation. Histopathology 1996;29:369-74

62 Clement PB, Scully RE. Endometrial stromal sarcomas of the uterus with extensive endometrioid glandular differentiation: a report of three cases that caused problems in differential diagnosis. Int J Gynecol Pathol 1992;11:163-73.

63 McCluggage WG, Cromie AJ, Bryson C, et al. Uterine endometrial stromal sarcoma with smooth muscle and glandular differentiation. J Clin Pathol 2001;54:481-3.

$64 \mathrm{Kim}$ YH, $\mathrm{Cho} \mathrm{H}$, Kyeom-Kim H, et al. Uterine endometrial stromal sarcoma with rhabdoid and smooth muscle differentiation. J Korean Med Sci 1996;1 1:88-93.

65 Rosty C, Genestie C, Blondon J, et al. Endometrial stromal tumor associated with rhabdoid phenotype and and zones of "sex cord-like" differentiation. Ann Pathol 1998;18:133-6.

66 Tanimoto A, Sasaguri T, Arima N, et al. Endometrial stromal sarcoma of the uterus with rhabdoid features. Pathol Int 1996:46:231-7.

67 Lloreta J, Prat J. Endometrial stromal nodule with smooth and skeletal muscle components simulating stromal sarcoma. Int J Gynecol Pathol 1992;1 1:293-8.

68 Baker PM, Moch H, Oliva E. Unusual morphologic features of endometrial stromal tumors: a report of 2 cases. Am J Surg Pathol 2005:29:1394-8.

69 Hauptmann S, Nadjari B, Kraus J, et al. Uterine tumor resembling ovarian sexcord tumor-a case report and review of the literature. Virchows Arch $2001 ; 439: 97-101$

70 Matias-Guiu X, Prat J. Alpha-inhibin immunostaining in diagnostic pathology. Adv Anat Pathol 1998:5:263-7.

71 Kommoss F, Oliva E, Bhan AK, et al. Inhibin expression in ovarian tumors and tumor-like lesions: an immunohistochemical study. Mod Pathol 1998;1 1:656-64.

72 Baker RJ, Hildebrandt RH, Rouse RV, et al. Inhibin and CD99 (MIC2) expression in uterine stromal neoplasms with sex-cord-like elements. Hum Pathol 1999;30:671-9.

73 Krishnamurthy S, Jungbluth AA, Busam KJ, et al. Uterine tumors resembling ovarian sex-cord tumors have an immunophenotype consistent with true sex-cord differentiation. Am J Surg Pathol 1998;22:1078-82.

74 De Leval L. Uterine tumors resembling ovarian sex-cord tumors: a study of 14 cases showing a diverse phenotypic profile. Am J Surg Pathol 2006;30:319-27.

75 Hillard JB, Malpica A, Ramirez PT. Conservative management of a uterine tumor resembling an ovarian sex cord-stromal tumor. Gynecol Oncol 2004;92:347-52.

76 Reich O, Regaver S, Urdl W, et al. Expression of oestrogen and progesterone receptors in low-grade endometrial stromal sarcomas. Br J Cancer 2000;82:1030-4.

77 Chu MC, Mor G, Lim C, et al. Low-grade endometrial stromal sarcoma: hormonal aspects. Gynecol Oncol 2003;90:170-6.

78 Reich 0, Regaver S. Aromatase expression in low-grade endometrial stromal sarcomas: an immunohistochemical study. Mod Pathol 2004;17:104-8.

79 Klein WM, Kurman RJ. The lack of expression of c-kit protein (CD117) in mesenchymal tumors of the uterus and ovary. Int J Gynecol Pathol 2002;22:181-4.

80 Geller MA, Argenta P, Bradley W, et al. Treatment and recurrence patterns in endometrial stromal sarcomas and the relation to c-kit expression. Gynecol Oncol 2004;95:632-6.

81 Leath CA 3rd, Straughn JM Jr, Conner MG, et al. Immunohistochemical evaluation of the c-kit proto-oncogene in sarcomas of the uterus: a case series. J Reprod Med 2004;49:71-5

82 Dal Cin P, Aly MS, De Wever I, et al. Endometrial stromal sarcoma $t(7 ; 17)(\mathrm{p} 15-$ $21 ; q 12-21)$ is a nonrandom chromosome change. Cancer Genet Cytogenet 1992;63:43-6.

83 Koontz JI, Soreng AL, Nucci M, et al. Frequent fusion of the JAZF1 and JJAZ1 genes in endometrial stromal tumors. Proc Natl Acad Sci USA $2001 ; 98: 6348-53$

84 Nucci MR, Harburger D, Koonte J, et al. Molecular analysis of the JAZF1-JJAZ gene fusion by RT-PCR and fluorescence in situ hybridization in endometrial stromal neoplasms. Am J Surg Pathol 2007;31:65-70.

85 Huang HY, Ladanyi M, Soslow RA. Molecular detection of JAZF1-JJAZ1 gene fusion in endometrial stromal neoplasms with classic and variant histology: evidence for genetic heterogeneity. Am J Surg Pathol 2004;28:224-32.

86 Oliva E, De Leval L, De Ceuninck C, et al. Interphase FISH detection of JAZF1JJAZ1 gene fusion in endometrial stromal tumors with smooth muscle differentiation. Mod Pathol 2006. In press. 